\title{
"Future Earth": Nigmatulin Hypothesis and Dynamic Model of Radiation Field of Ocean-Atmosphere System
}

\author{
Tamara Sushkevich ${ }^{1, *}$, Sergey Strelkov ${ }^{1}$, and Svetlana Maksakova ${ }^{1}$ \\ ${ }^{1}$ Keldysh Institute of Applied Mathematics of RAS, Department Dynamic systems, RU-125047, Moscow, Russia
}

\begin{abstract}
The United Nations has proclaimed a Decade of Ocean Science for Sustainable Development (2021-2030) to support efforts to reverse the cycle of decline in ocean health and gather ocean stakeholders worldwide behind a common framework that will ensure ocean science can fully support countries in creating improved conditions for sustainable development of the Ocean. The marine realm is the largest component of the Earth's system that stabilizes climate and support life on Earth and human well-being. Scientific understanding of the ocean's responses to pressures and management action is fundamental for sustainable development. Planet Earth is a natural example of a dynamic system with nonlinear processes that is in continuous change. The Earth's radiation field is a single physical field (electromagnetic radiation) and the unifying factor of the Earth dynamical system. The Earth's climate system is a natural environment that includes the atmosphere, the hydrosphere (oceans, seas, lakes, rivers), the cryosphere (land surface, snow, sea and mountain ice, etc.), and the biosphere that unites all living things. According to the hypothesis of R.I. Nigmatulin "Ocean is a dictator of climate". H2O and CO2 are competing climate influences. In this article, we propose original author's mathematical models for radiation blocks with hyperspectral data on absorption by atmospheric components. The new models are based on the development of the theory of the optical transfer operator and the method of influence functions in the theory of radiation transfer and Boltzmann equations, as well as the iterative method of characteristics with iteration convergence accelerations.
\end{abstract}

\section{Introduction}

The United Nations has proclaimed a Decade of Ocean Science for Sustainable Development (2021-2030) to support efforts to reverse the cycle of decline in ocean health and gather ocean stakeholders worldwide behind a common framework that will ensure ocean science can fully support countries in creating improved conditions for sustainable development of the Ocean [1]. The marine realm is the largest component of the Earth's system that stabilizes climate and support life on Earth and human well-being. Scientific understanding of the ocean's responses to pressures and management action is fundamental for sustainable development. Ocean observations and research are also essential to predict the consequences of change, design mitigation and guide adaptation. As mandated by the UN General Assembly the Intergovernmental Oceanographic Commission (IOC) of UNESCO will coordinate the Decade's preparatory process, inviting the global ocean community to plan for the next ten years in ocean science and technology.

From November 30 to December 12, 2015, The 21st Conference of the Parties Climate Summit (COP21) was held in Paris, where the heads of more than 150 States and about 40 thousand researchers. On December 12, 2015, the "Paris Agreement under the United Nations Framework Convention on Climate Change" ("Paris Agreement”) was adopted, which, after signing from
April 22, 2016 to April 21, 2017, entered into force instead of "Kyoto Protocol".

The global challenge to the Convention "Agenda 21", adopted at the United Nations Conference on Environment and Development, Rio de Janeiro, June 314, 1992, the Resolution "Transforming our world: the 2030 Agenda for Sustainable Development”, adopted by the General Assembly on 25 September 2015, and the super-large project, which has no analogues in scale and significance for all of humanity in world history is the World Global Scientific Program "Future Earth" (Program).

The fundamental basis of the Program to ensure the sustainable development and existence of human society and States on the planet were laid in the 20th century: this is the unprecedented flowering of mathematics, the invention of the computer, the Internet and the extraordinary earlier information, communication and digital technologies, as well as the exit of man into space, exploration of space, the development of the global observation systems and remote sensing of the Earth, objects and processes on the basis of the "light technologies" when creating the "Nuclear missile shield" and implementation of the "Atomic" and "Space" projects.

1951 was a key year in the life of M.V. Keldysh [2]. From the Resolution of the USSR Council of Ministers No. 1552-774ss / op of May 9, 1951 “On works on RDS-

Corresponding author: tamaras@keldysh.ru 
6T” (Atomic project: documents and materials. Vol. 3. Book 1. 2008. pp. 396-403.):

"4. Oblige the First Main Directorate under the USSR Ministers Council to organize a mathematical section (section 7) as part of the Scientific and technical council for scientific guidance on the development of designs, high-speed computers, as well as methods of their operation in the composition of: academician M.V. Keldysh - section president; section members: academician I.G. Petrovsky, academician S.L Sobolev, corresponding member N.N. Bogolyubov, corresponding member A.N. Tikhonov; section members (for computers): academician M.A. Lavrent'ev, corresponding member S.A. Lebedev, engineer Yu. Bazilevsky, engineer M.A. Lesechko.

Assign to Section 7 the consideration of plans for research, experimental and design work, as well as projects of mathematical machines and work plans of organizations performing calculation work on the subject of the First Main Directorate under the USSR Ministers Council."

In the year of the 75th anniversary of the Soviet “Atomic Project" (1945-2020), it is important to note the creation of the world's strongest Russian mathematical school, which has survived and still occupies a leading position in world science. The foundations of applied mathematics were laid in the middle of the 20th century under the leadership of M.V. Keldysh - the Chief Mathematician responsible for computers and calculations in the "Atomic Project", "Space Project" and the "Nuclear Missile Shield" project ("Three Projects"). In 1953, the Chief Cosmonautics Theorist M.V. Keldysh $[3,4]$ created the world's first Institute of applied mathematics of the USSR Academy of Sciences (Keldysh Institute) for the implementation of "Three Projects" using a computer.

At the stage of space exploration and the formation of space research and Earth remote sensing from space, the United States invited mathematicians and specialists in the theory of radiation transfer from different countries to NASA. However, the mathematical scientific school in the United States was not formed. In recent decades, in the centers of NASA and the United States, the EU, China, France, Japan and other countries, with the presence of supercomputers, researchers are passionate about information technology and data processing ("computer sciences").

During the "nuclear" threats in the 20th century, the scientists saved the planet for humanity. And this experience is now in great demand, since the threat of the global changes on the planet is already obvious. The Program "Future Earth" is a natural, comprehensive stage in the development of science, technology and society, aimed at ensuring life on planet Earth. The priceless lessons must be drawn from these successful projects. They have contributed to the development of mathematics, physics, chemistry, theory and applications of Earth sciences, and "computer sciences". The USSR pursued a real peace-loving policy and did not rush to develop means for delivering "nuclear strikes".

The recent UNESCO General Conference has adopted a resolution to proclaim 2022 as the
International Year of Basic Sciences for Development (IYBSD). This is great news, as the Year would help highlight the crucial role of basic sciences for sustainable development, and emphasize their contributions to the implementation of the 2030 Agenda and achievement of the Sustainable Development Goals (SDGs).

This article focuses on the problems of the planet protection associated with the Earth's radiation field, the radiation transfer in natural environments, the role of solar and intrinsic radiation in the global Earth Climatic System (ECS) and Earth remote sensing (ERS). The radiation forcing, the role of the humidity in different phase states, the contribution of the ocean, and the radiation exchange between the atmosphere and the ocean are highlighted. These problems require deep study.

\section{Nigmatulin hypothesis and the radiation factor}

In the works of the great domestic scientists V.I. Vernadsky and N.N. Moiseev actually formulated the main provisions of the idea of sustainable development. N.N. Moiseev, perhaps, was the first to move from the geographical concepts of "climate" and "ecology" to the planetary problems of climate and sustainable development of the planet and created the scientific basis for the study of ECS and the co-evolution of nature and society.

"The ocean and short-period climate variability" - on such problems since the end of 1970 and in the 1980s the large-scale studies were conducted in the framework of the Russian program "Cuts" under the general scientific leadership of academicians G.I. Marchuk and A.S. Sargsyan. Initially, this program was only of Russian affiliation, but then part of it became part of the International Program World Ocean Circulation Experiment (WOCE).

The Earth Climate System is a natural environment, including the atmosphere, the hydrosphere (oceans, seas, lakes, rivers), the cryosphere (land surface, snow, sea and mountain ice, etc.), the biosphere that unites all living things. For quantitative assessments of the significance of different climate-forming factors, depending on solar and intrinsic radiation, we introduced a special characteristic of ECS - radiation exposure (forcing). According to expert estimates, from $40 \%$ to $60 \%$ of the impact of various factors on the evolution of climate falls on radiation forcing.

On the one hand, the solar radiation - a source of energy on Earth - is one of the integral factors of life support for the human, animal and plant world on Earth, as well as one of the determining components of the Earths ecosystem and biosphere, the behavior of which is characterized by the interaction of the individual components with the manifestation of synergism, i. e. feedbacks, which sometimes lead to mutual reinforcement of various processes.

On the other hand, the solar radiation field affects the mechanisms of variability (dynamic processes: circulation, convection, turbulent transport; radiation and 
photochemical processes) of the geophysical, meteorological, climatic conditions of the Earth, which have complex nonlinear relationships that make it difficult to predict possible effects, estimate their magnitude and significance.

On the third hand, the electromagnetic radiation detected by various means is the main source of information about the structure and physical properties of planetary atmospheres, surfaces, the ocean, the biosphere during conservative remote sensing. In active systems, a laser or spotlight can be used as an insolation source.

The physical picture of the climate of the ocean-landatmosphere system was presented by A.S. Monin in the review [5]. R.I. Nigmatulin called the expert article "Ocean - the dictator of climate" [6]. More study of the $\mathrm{H} 2 \mathrm{O}$ and $\mathrm{CO} 2$ cycle is needed. To test his realistic hypotheses for explaining the mechanisms of the most complex interaction of the atmosphere with clouds and the ocean and their influence on climate, it is important to be able to model the formation of clouds and to separate the contributions of the atmosphere and the ocean to the total radiation field of the Earth, recorded from space.

Nigmatulin hypothesis:

"The world ocean occupies more than $70 \%$ of the planet's surface. Solar radiation is the source of energy on Earth. It is absorbed and accumulated by the ocean, then re-emitted into the long-wave spectrum. This radiation is already absorbed by greenhouse gases carbon dioxide and water vapor - and warms the atmosphere. And water vapor in the atmosphere is two orders of magnitude more than carbon dioxide. However, nature provides a compensatory mechanism... So far, this is a hypothesis: as the vapor content in the air increases, cloud cover should increase, which means that the reflection of solar radiation by clouds increases. There is a negative feedback..."

This is the most complex natural radiation process on a global scale, on which the future of the planet depends. To research radiation influence of the oceans on the Earth's evolution requires international cooperation with the involvement of integrated observing systems of radiation processes under water, at sea and from space with the support of supercomputer simulation [7].

The study of the hypothesis is carried out on the basis of a "scenario" approach. The complex model contains four main blocks:

1) solar radiation transfer in the ocean-atmosphere system,

2) evaporation from the ocean surface and formation of a humidity field in the atmosphere,

3) the formation of clouds,

4) transfer of solar radiation in the ocean-atmosphere system with clouds.

In this article, we propose original author's mathematical models for radiation blocks [8-10] with hyperspectral data on absorption by atmospheric components $[11,12]$. The new models are based on the development of the theory of the optical transfer operator and the method of influence functions in the theory of radiation transfer and Boltzmann equations [8, 9], as well as the iterative method of characteristics with iteration convergence accelerations [10].

At present, numerical models of radiation transfer in the ocean-atmosphere system (OAS) and mechanisms of forming radiation fields in OAS with allowance for short-wavelength radiation transfer between the media continue to attract interest. Seven foreign models were compared to one another with the support of international foundations [13]. Five of the models were implemented by the Monte Carlo method; another one, by the method of invariant imbedding; and the remaining one, by the method of discrete coordinates, or more exactly, by the method of spherical harmonics (DISORT program) [14]. The latter two models used a preliminary Fourier expansion in terms of azimuth. All the models are one-dimensional in space and include an air-water Fresnel interface. The numerical models that most researchers use to calculate radiation characteristics are presented in report of the "Radiation commission" [15].

\section{Model of radiation transfer in the ocean-atmosphere system and the influence functions method}

Solar-radiation propagation in the ocean-atmosphere system are modeled as radiation transfer in a twomedium plane-parallel layer non-uniform in height and with the air-water interface reflecting and transmitting radiation by the Fresnel law.

\subsection{Formulation of the mathematical problem}

A one-dimensional planar model is considered of the atmosphere-ocean system illuminated by a monodirectional parallel flux of solar radiation. A new approach is proposed to radiation transfer modeling and daylight background formation for the atmosphere that is represented as a heterogeneous two-layer system each layer of which is described by different optical characteristics. The influence functions of each layer are determined by solutions of the radiation transfer boundary problem with an external mono-directional wide flux while the contribution of multiple scattering and absorption in the layer is taking into account.

The direction of radiation transfer in the atmosphere is described by the vector $s=(\mu, \varphi)$, $\mu=\cos \vartheta \in[-1,1]$, on the unit sphere $\Omega=[-1,1] \times[0,2 \pi]$, where $\vartheta \in[0, \pi]$ is the zenith angle measured from the positive direction of the $z$-axis and $\varphi \in[0,2 \pi]$ is the azimuth. The value $\varphi=0$ is attributed to the plane of the sun's vertical passing through the $z$ axis and the direction $s_{0}=\left(\mu_{0}, \varphi_{0}\right)$ of solar flux incidence upon the upper boundary of the layer $Z=0$; $\mu_{0}=\cos \vartheta_{0} \in(0,1] ; \quad \vartheta_{0} \in[0, \pi / 2) ; \quad \varphi_{0}=0 ; \quad$ and $s_{0}^{-}=\left(-\mu_{0}, \varphi_{0}\right)$.

If $s=(\mu, \varphi)$, then $-s=(-\mu, \varphi+\pi)$. 
For the downward radiation

$$
s=s^{+}=\left(\mu^{+}, \varphi^{+}\right) \in \Omega^{+}=\{(\mu, \varphi): \mu \geq 0\}
$$

for the upward radiation

$$
s=s^{-}=\left(\mu^{-}, \varphi^{-}\right) \in \Omega^{-}=\{(\mu, \varphi): \mu \leq 0\} ;
$$

and $\Omega=\Omega^{+} \cup \Omega^{-}$.

The direction of transfer in the ocean is defined by the vector $\quad \tilde{s}=(\tilde{\mu}, \tilde{\varphi}), \quad \tilde{\mu}=\cos \tilde{\vartheta} \in[-1,1]$, $\tilde{\varphi} \in[0,2 \pi], \tilde{\varphi}=\varphi_{0}=0, \tilde{s} \in \Omega ; \tilde{s}^{+}=\left(\tilde{\mu}^{+}, \tilde{\varphi}^{+}\right) \in \Omega^{+} ;$ and $\tilde{s}^{-}=\left(\tilde{\mu}^{-}, \tilde{\varphi}^{-}\right) \in \Omega^{-}$.

The direction of the ray $s^{+}$that is incident upon the interface from the side of the atmosphere and travels into the ocean after refraction is described by the vector

$$
\bar{S}=\bar{S}^{+}=\left(\bar{\mu}^{+}, \bar{\varphi}^{+}\right) \in \Omega_{c r i t}^{+}=\left[\mu_{c r i t}, 1\right] \times[0,2 \pi] .
$$

The direction

$$
\bar{S}=\bar{S}^{-}=\left(\bar{\mu}^{-}, \bar{\varphi}^{-}\right) \in \Omega_{c r i t}^{-}=\left[-1,-\mu_{c r i t}\right] \times[0,2 \pi]
$$

corresponds to the ray that is incident upon the interface from the side of the ocean and assumes the direction $S^{-}$ in the atmosphere after refraction.

The interaction of radiation with the Fresnel boundary is governed by the geometrical-optics laws. The reflection law determines the relationship between the directions of incident $S=(\vartheta, \varphi)$ and reflected $s^{\prime}=\left(\vartheta^{\prime}, \varphi^{\prime}\right)$ rays: $\mu^{\prime}=-\mu, \varphi^{\prime}=\varphi$. The refraction law controls the relationship between the directions of incident $S$ and refracted $\bar{S}=(\bar{\vartheta}, \bar{\varphi})$ rays:

$$
n_{1} \sin \vartheta=n_{2} \sin \bar{\vartheta}, \sin \bar{\vartheta}=\sin \vartheta / n, n=n_{2} / n_{1}, \quad \varphi=\bar{\varphi}
$$

where $n_{1}$ and $n_{2}$ are the refractive coefficients of the media. In accordance with the refraction law, we have

$$
\bar{\mu}=k \mu / n, \quad k=\sqrt{1+\left(n^{2}-1\right) / \mu^{2}}, \mu_{c r i t}=\sqrt{1-1 / n^{2}} .
$$

If $n=1.33$, then $\vartheta_{\text {crit }} \approx 48.75^{\circ}$.

The Fresnel coefficient is

$$
r(\vartheta)=r(\bar{\vartheta})=\frac{1}{2}\left[\frac{\sin ^{2}(\bar{\vartheta}-\vartheta)}{\sin ^{2}(\bar{\vartheta}+\vartheta)}+\frac{\operatorname{tg}^{2}(\bar{\vartheta}-\vartheta)}{\operatorname{tg}^{2}(\bar{\vartheta}+\vartheta)}\right] .
$$

For the normal incidence of radiation, $\bar{\vartheta}=\vartheta=0$ and

$$
r(\vartheta)=r(\bar{\vartheta})=(n-1)^{2} /(n+1)^{2} \text {. }
$$

The directions $\quad \tilde{S}^{-} \in \Omega^{-} \backslash \Omega_{\text {crit }}^{-} \quad$ with $\tilde{\mu}^{-} \in\left(-\mu_{\text {crit }}, 0\right)$ in the ocean form the region of total internal reflection when $r(\tilde{\vartheta})=1$. The directions $\tilde{S}^{+} \in \Omega^{+} \backslash \Omega_{\text {crit }}^{+}$with $\tilde{\mu}^{+} \in\left(0, \mu_{\text {crit }}\right)$ in the ocean lie in the shadow region, which is not illuminated by the light arriving from the atmosphere.

The spatial and angular distribution of solar-radiation intensity $\Phi(z, \mu, \varphi)$ in the two-medium OAS is described by the boundary-value problem

$$
\begin{gathered}
K \Phi=0,\left.\Phi\right|_{t}=\pi S_{\lambda} \delta\left(s-s_{0}\right),\left.\Phi\right|_{b}=R_{H} \Phi \\
\left.\Phi\right|_{d 1}=R_{1} \Phi+T_{21} \Phi,\left.\Phi\right|_{d 2}=R_{2} \Phi+T_{12} \Phi
\end{gathered}
$$

with the linear operators of transfer and collisions:

$$
D \equiv \mu \frac{\partial}{\partial z}+\sigma_{\text {tot }}(z),
$$

$$
\begin{gathered}
S \Phi \equiv \sigma_{s c}(z) \int_{\Omega} \gamma\left(z, s, s^{\prime}\right) \Phi\left(z, s^{\prime}\right) d s^{\prime} \\
K \equiv D-S
\end{gathered}
$$

where $d s^{\prime}=d \mu^{\prime} d \varphi^{\prime} ; \sigma_{\text {tot }}(z)$ and $\sigma_{s c}(z)$ are the vertical profiles of the extinction and scattering coefficients, respectively; and $\gamma\left(z, s, s^{\prime}\right)$ is the scattering phase function. The source of radiation is an external parallel solar flux to the upper bound $z=0$. At the level $z=h$ within the layer $z \in[0, H]$, the interface between two media is located.

For the Fresnel air-water interface, the reflection operators $R_{1}, R_{2}$ and the transmittance operators $T_{12}$, $T_{21}$ act according to the following rules:

$$
\begin{gathered}
{\left[R_{1} \Phi\right]\left(s^{-}\right)=\Phi\left(h, s^{+}\right) r\left(\mu^{+}\right),} \\
{\left[R_{2} \Phi\right]\left(\tilde{s}^{+}\right)=\Phi\left(h, \tilde{s}^{-}\right) r\left(\left|\tilde{\mu}^{-}\right|\right),} \\
{\left[T_{12} \Phi\right]\left(\bar{s}^{+}\right)=\Phi\left(h, s^{+}\right)\left[1-r\left(\mu^{+}\right)\right] n^{2},} \\
{\left[T_{21} \Phi\right]\left(s^{-}\right)=\Phi\left(h, \bar{s}^{-}\right)\left[1-r\left(\left|\bar{\mu}^{-}\right|\right)\right] / \mathrm{n}^{2} .}
\end{gathered}
$$

For the reflecting bottom,

$\left[R_{H} \Phi\right]\left(\tilde{S}^{-}\right)=\int_{\Omega^{+}} \Phi\left(H, \tilde{s}^{+}\right) q_{H}\left(\tilde{s}^{-}, \tilde{s}^{+}\right) d \tilde{s}^{+}$

The boundary conditions are written using the sets: 


$$
\begin{aligned}
& t=\left\{(z, s): \quad z=0, \quad s=s^{+} \in \Omega^{+}\right\}, \\
& b=\left\{(z, \tilde{s}): \quad z=H, \quad \tilde{s}=\tilde{s}^{-} \in \Omega^{-}\right\}, \\
& d 1=\left\{(z, s): \quad z=h, \quad s=s^{-} \in \Omega^{-}\right\}, \\
& d 2=\left\{(z, \tilde{s}): \quad z=h, \quad \tilde{s}=\tilde{s}^{+} \in \Omega^{+}\right\},
\end{aligned}
$$

whose designations correspond to the first letters of the words top, bottom, and dividing.

For brevity and clarity, we will use the indices " $s$ " (singular), “ $c$ " (continued), “ $d$ " (diffuse), the upper index " 0 ” for the direct attenuated radiation, " $a$ ” (atmosphere), and "ok" (ocean).

We will solve the problem (1) by the method of influence functions on the basis of an OTO model. Consider several ways of structuring the solution of the initial problem (1), which correspond to different OTO approximations and make it possible to thoroughly study the mechanisms of forming the radiation field in the OAS.

The problem that describes the transfer of the direct solar flux in the OAS and includes only attenuation in the atmosphere and ocean and only one act of interaction with the interface:

$$
\begin{gathered}
D \Phi^{0}=0,\left.\Phi^{0}\right|_{t}=\pi S_{\lambda} \delta\left(s-s_{0}\right),\left.\Phi^{0}\right|_{b}=0, \\
\left.\Phi^{0}\right|_{d 1}=R_{1} \Phi^{0},\left.\Phi^{0}\right|_{d 2}=T_{12} \Phi^{0}
\end{gathered}
$$

can be solved in an explicit form if the Fresnel law is assumed to be valid at the interface. In the atmosphere,

$$
\begin{aligned}
& \Phi^{0}\left(s_{0} ; z, s^{+}\right)=\Phi^{0}\left(s_{0} ; z, s_{0}\right) \delta\left(s^{+}-s_{0}\right), \\
& \Phi^{0}\left(s_{0} ; z, s_{0}\right)=\pi s_{\lambda} \exp \left[-\tau(z) / \mu_{0}\right] .
\end{aligned}
$$

The direct radiation reflected from the interface into the atmosphere in the direction $s_{0}^{-}$can be written as

$$
\begin{gathered}
\Phi^{0}\left(s_{0} ; z, s^{-}\right)= \\
=\Phi^{0}\left(s_{0} ; h, s^{-}\right) \exp \left\{-[\tau(h)-\tau(z)] / \mu_{0}\right\}, \\
\Phi^{0}\left(s_{0} ; h, s^{-}\right)=E_{a}^{s}\left(s_{0} ; s^{-}\right) \equiv R_{1} \Phi^{0}= \\
=\Phi^{0}\left(s_{0} ; h, s_{0}\right) r\left(\mu_{0}\right) \delta\left(s^{-}-s_{0}^{-}\right) .
\end{gathered}
$$

The direct radiation that penetrates into the ocean after refraction is written as

$$
\begin{gathered}
\Phi^{0}\left(\bar{s}_{0} ; z, \bar{S}^{+}\right)= \\
=\Phi^{0}\left(\bar{s}_{0} ; h, \bar{S}^{+}\right) \exp \left\{-[\tau(z)-\tau(h)] / \bar{\mu}_{0}\right\},
\end{gathered}
$$

$$
\begin{aligned}
& \Phi^{0}\left(\bar{S}_{0} ; h, \bar{S}^{+}\right)=E_{o k}^{s}\left({\overline{S_{0}}}_{0} ; \bar{S}^{+}\right) \equiv T_{12} \Phi^{0}= \\
& =\Phi^{0}\left(s_{0} ; h, s_{0}\right)\left[1-r\left(\mu_{0}\right)\right] n^{2} \delta\left({\overline{S_{0}}}-\bar{S}^{+}\right) .
\end{aligned}
$$

The optical thickness of the layer $[0, z]$ is described by the formula

$$
\tau(z)=\int_{0}^{z} \sigma_{t o t}\left(z^{\prime}\right) d z^{\prime}
$$

\subsection{The optical transfer operators and method of influence functions}

For estimation of the influence of ocean radiation in the layer $z \in[0, H]$, we will consider the general boundary-value problem for the OAS (GBPOAS) when at least one of the conditions $T_{12} \not \equiv 0, T_{21} \not \equiv 0$ is satisfied:

$$
\begin{aligned}
& K \Phi_{o k}=0,\left.\Phi_{o k}\right|_{t}=0,\left.\Phi_{o k}\right|_{b}=0, \\
& \left.\Phi_{o k}\right|_{d 1}=\eta\left(R_{1} \Phi_{o k}+T_{21} \Phi_{o k}+E_{a}\right), \\
& \left.\Phi_{o k}\right|_{d 2}=\eta\left(R_{2} \Phi_{o k}+T_{12} \Phi_{o k}+E_{o k}\right) .
\end{aligned}
$$

Here, $E_{a}=E_{a}^{s}+E_{a}^{d}$ and $E_{o k}=E_{o k}^{s}+E_{o k}^{d}$ are the source functions of radiation, which generally involve the singular $E_{a}^{s}, E_{o k}^{s}$ and diffuse $E_{a}^{d}, E_{o k}^{d}$ components, and the parameter $\eta$ takes into account one act of radiation transmission through the interface.

Let us introduce the two-component algebraic vectors

$$
\begin{gathered}
\boldsymbol{\Theta}=\left\{\Theta_{a}, \Theta_{o k}\right\}, \quad \boldsymbol{\Phi}_{o k}=\left\{\Phi^{a}, \Phi^{o k}\right\}, \\
\boldsymbol{\Phi}_{n}=\left\{\Phi_{a, n}, \Phi_{o k, n}\right\}, \quad \mathbf{E}=\left\{E_{a}, E_{o k}\right\} .
\end{gathered}
$$

The solution of the GBPOAS (7) is sought in the form of a series of regular perturbations

$$
\boldsymbol{\Phi}_{o k}=\sum_{n=1}^{\infty} \eta^{n} \boldsymbol{\Phi}_{n},
$$

whose components satisfy the recurrence system of the first boundary-value problems for the atmospheric layer $z \in[0, h]$ (FBPA) and the oceanic layer $z \in[h, H]$ (FBPO):

$$
\begin{gathered}
K \Phi_{a, n}=0,\left.\Phi_{a, n}\right|_{t}=0,\left.\Phi_{a, n}\right|_{d 1}=F_{a, n-1} ; \\
K \Phi_{o k, n}=0,\left.\Phi_{o k, n}\right|_{b}=0,\left.\Phi_{o k, n}\right|_{d 2}=F_{o k, n-1}
\end{gathered}
$$


with the initial source approximations $F_{a, 0} \equiv E_{a}$, $F_{o k, 0} \equiv E_{o k}$.

The solutions in all $n$-approximations are found as linear functionals:

$$
\Phi_{a, n}=\left(\Theta_{a}, F_{a, n-1}\right), \Phi_{o k, n}=\left(\Theta_{o k}, F_{o k, n-1}\right)
$$

the sources are found:

$$
F_{a, n}=R_{1} \Phi_{a, n}+T_{21} \Phi_{o k, n}, F_{o k, n}={ }_{2} \Phi_{o k, n}+T_{12} \Phi_{a, n} .
$$

If $E_{a}\left(s^{-}\right)$is the irradiance of the interface from the side of the atmosphere, then for $z \in[0, h], s \in \Omega$ we have the functional

$$
\left(\Theta_{a}, E_{a}\right)(z, s)=\frac{1}{2 \pi} \int_{\Omega^{-}} \Theta_{a}\left(s_{h}^{-} ; z, s\right) E_{a}\left(s_{h}^{-}\right) d s_{h}^{-} .
$$

If $E_{o k}\left(\tilde{S}^{+}\right)$is the irradiance of the interface from the side of the ocean, then for $z \in[h, H]$, or $\tilde{Z}=Z-h \in[0, H-h], \tilde{s} \in \Omega$ we have the functional

$$
\left(\Theta_{o k}, E_{o k}\right)(\tilde{z}, \tilde{s})=\frac{1}{2 \pi} \int_{\Omega^{+}} \Theta_{o k}\left(\tilde{s}_{h}^{+} ; \tilde{z}, \tilde{s}\right) E_{o k}\left(\tilde{s}_{h}^{+}\right) d \tilde{s}_{h}^{+} .
$$

The kernels of the functionals are the atmosphere IF $\Theta_{a}\left(s_{h}^{-} ; z, s\right)$, which represents the solution of the FBPA with the parameter $s_{h}^{-} \in \Omega^{-}$:

$$
K \Theta_{a}=0,\left.\Theta_{a}\right|_{t}=0,\left.\Theta_{a}\right|_{d 1}=\delta\left(s-s_{h}^{-}\right)
$$

and the ocean IF $\Theta_{o k}\left(\tilde{S}_{h}^{+} ; \tilde{z}, \tilde{S}\right)$, which represents the solution of the FBPO with the parameter $\tilde{s}_{h}^{+} \in \Omega^{+}$:

$$
K \Theta_{o k}=0,\left.\Theta_{o k}\right|_{b}=0,\left.\Theta_{o k}\right|_{d 2}=\delta\left(\tilde{s}-\tilde{s}_{h}^{+}\right) .
$$

These problems (9) and (10) are similar to problems where the radiation source is a wide unidirectional (for example, solar) flux.

For the atmosphere, a set of atmosphere IFs with the parameter

$$
s_{h}^{-}=\left\{\left(\mu_{h}^{-}, \varphi_{h}^{-}\right): \varphi_{h}^{-}=0, \mu_{h}^{-} \in[-1,0)\right\}
$$

are calculated as a superposition: $\Theta_{a}=\Theta_{a}^{s}+\Theta_{a}^{d}$. The singular component describes the transfer of direct radiation from the interface:

$$
\Theta_{a}^{s}\left(s_{h}^{-} ; z, s^{-}\right)=
$$

$$
=\exp \left\{-[\tau(h)-\tau(z)] /\left|\mu_{h}^{-}\right|\right\} \delta\left(s^{-}-s_{h}^{-}\right) .
$$

The continued component represents the solution of the FBPA with allowance for multiple scattering

$$
K \Theta_{a}^{d}=\hat{S} \Theta_{a}^{s},\left.\Theta_{a}^{d}\right|_{t}=0,\left.\Theta_{a}^{d}\right|_{d 1}=0
$$

For the ocean, a set of ocean IFs with the parameter

$$
\tilde{s}_{h}^{+}=\left\{\left(\tilde{\mu}_{h}^{+}, \tilde{\varphi}_{h}^{+}\right): \tilde{\varphi}_{h}^{+}=0, \tilde{\mu}_{h}^{+} \in(0,1]\right\}
$$

as a superposition: $\Theta_{o k}=\Theta_{o k}^{s}+\Theta_{o k}^{d}$.

The singular component corresponds to the transmission of direct radiation from the interface:

$$
\Theta_{o k}^{s}\left(\tilde{S}_{h}^{+} ; \tilde{z}, \tilde{s}^{+}\right)=\exp \left[-\tau(\tilde{z}) / \tilde{\mu}_{h}^{+}\right] \delta\left(\tilde{s}^{+}-\tilde{s}_{h}^{+}\right) .
$$

The continued component represents the solution of the FBPO with allowance for multiple scattering

$$
K \Theta_{o k}^{d}=\hat{S} \Theta_{o k}^{s},\left.\Theta_{o k}^{d}\right|_{b}=0,\left.\Theta_{o k}^{d}\right|_{d 2}=0 .
$$

The diffuse components of the IFs of the atmosphere and ocean are calculated as the solutions of problems (11) and (12) obtained by the known methods in the approximations corresponding to the optical parameters of the two media.

Using the algebraic vector of the IFs of the atmosphere and ocean, we introduce a vector operation describing one act of radiation-interface interaction and taking into account multiple scattering in both media:

$$
\begin{gathered}
{[G \mathbf{E}](s) \equiv P(\Theta, \mathbf{E})=} \\
=\left[\begin{array}{c}
R_{1}\left(\Theta_{a}, E_{a}\right)+T_{21}\left(\Theta_{o k}, E_{o k}\right) \\
T_{12}\left(\Theta_{a}, E_{a}\right)+R_{2}\left(\Theta_{o k}, E_{o k}\right)
\end{array}\right], \\
P \equiv\left[\begin{array}{cc}
R_{1} & T_{21} \\
T_{12} & R_{2}
\end{array}\right],
\end{gathered}
$$

where $(\Theta, E)=\left\{\left(\Theta_{a}, E_{a}\right),\left(\Theta_{o k}, E_{o k}\right)\right\}$ is the vector functional. Two successive $n$-approximations of series (8) are related by the recurrence formula

$$
\Phi_{n}=\left(\Theta, P \Phi_{n-1}\right) .
$$

An asymptotically exact solution of the GBPOAS (7) is obtained in the form of a linear functional, optical transfer operator of the OAS,

$$
\Phi_{o k}=(\Theta, \mathbf{Z})
$$

where the "scenario" $\mathbf{Z}=\left\{Z_{a}, Z_{o k}\right\}$ of the irradiance pattern at the interface 


$$
\mathbf{Z} \equiv \sum_{n=0}^{\infty} G^{n} \mathbf{E}=\sum_{n=0}^{\infty} P \Phi_{n}
$$

is the sum of the Neumann series in multiplicity of radiation transmission through the interface with the contribution of multiple scattering in both media taken into account.

The following identical representations are valid:

$$
\begin{gathered}
R_{1}\left(\Theta_{a}, E_{a}\right)=\left(R_{1} \Theta_{a}, E_{a}\right), \\
R_{2}\left(\Theta_{o k}, E_{o k}\right)=\left(R_{2} \Theta_{o k}, E_{o k}\right), \\
T_{12}\left(\Theta_{a}, E_{a}\right)=\left(T_{12} \Theta_{a}, E_{a}\right), \\
T_{21}\left(\Theta_{o k}, E_{o k}\right)=\left(T_{21} \Theta_{o k}, E_{o k}\right) .
\end{gathered}
$$

According to these identities, a more rapid calculation of the elements of the operator (13) can be performed by preliminarily determining $R_{1} \Theta_{a}, \quad R_{2} \Theta_{o k}, \quad T_{12} \Theta_{a}$, $T_{21} \Theta_{o k}$ from formulas (2) and (3).

If the ocean is regarded as the reflecting underlying surface, the contribution of radiation multiply reflected from the interface and multiply scattered in the atmosphere is described by the general boundary-value problem (GBPA) with $R_{1} \not \equiv 0$ (the parameter $\varepsilon$ takes into account an event of reflection from the interface):

$$
\begin{aligned}
& K \Phi_{a r}=0,\left.\Phi_{a r}\right|_{t}=0, \\
& \left.\Phi_{a r}\right|_{d 1}=\varepsilon\left(R_{1} \Phi_{a r}+E_{a r}\right) .
\end{aligned}
$$

The solution of the GBPA (14) is sought as the series

$$
\Phi_{a r}(z, s)=\sum_{n=1}^{\infty} \varepsilon^{n} \Phi_{n}(z, s),
$$

whose terms satisfy the recurrence system of the FBPA

$$
\begin{gathered}
K \Phi_{n}=0,\left.\Phi_{n}\right|_{t}=0,\left.\Phi_{n}\right|_{d 1}=F_{n-1}, \\
F_{n-1}=R_{1} \Phi_{n-1}, F_{0}=E_{a r}
\end{gathered}
$$

and are found as linear functionals with kernels representing the atmosphere IFs:

$$
\Phi_{n}=\left(\Theta_{a}, F_{n-1}\right) .
$$

If $E_{a r}\left(s^{-}\right)$is a fixed irradiance of the interface, then using the operation

$$
Q E_{a r} \equiv R_{1}\left(\Theta_{a}, E_{a r}\right)=\left(R_{1} \Theta_{a}, E_{a r}\right)
$$

one can describe both the corresponding contribution of multiple scattering in the atmosphere, which is included through the atmosphere IFs, and one act of radiation reflection from the underlying surface. An asymptotically exact solution of the GBPA (14) is determined as a linear functional - the optical transfer operator of the underlying surface

$$
\Phi_{a r}=\left(\Theta_{a}, Z_{a r}\right) .
$$

The full "scenario" of the irradiance pattern at the water surface is

$$
\begin{aligned}
& Z_{a r}\left(s^{-}\right) \equiv \sum_{n=0}^{\infty} Q^{n} E_{a r}= \\
& =\sum_{n=0}^{\infty} R_{1} \Phi_{n}=E_{a r}+R_{1} Y_{a r} .
\end{aligned}
$$

This Neumann series in multiplicity of radiation reflection from the water with allowance for multiple scattering in the air is expressed through the diffuse "irradiance" of the interface, which is caused by the backscattering of reflected radiation in the atmosphere:

$$
Y_{a r}\left(s^{+}\right)=\sum_{n=1}^{\infty} \Phi_{n}\left(h, s^{+}\right) .
$$

\subsection{Functional calculation}

In the general case, it is necessary to separate singular and continued diffuse components, because each functional can involve four components with different properties:

$$
\begin{gathered}
\left(\Theta_{a}, E_{a}\right)=\left(\Theta_{a}^{s}, E_{a}^{s}\right)+ \\
+\left(\Theta_{a}^{d}, E_{a}^{s}\right)+\left(\Theta_{a}^{s}, E_{a}^{d}\right)+\left(\Theta_{a}^{d}, E_{a}^{d}\right), \\
\left(\Theta_{o k}, E_{o k}\right)=\left(\Theta_{o k}^{s}, E_{o k}^{s}\right)+ \\
+\left(\Theta_{o k}^{d}, E_{o k}^{s}\right)+\left(\Theta_{o k}^{s}, E_{o k}^{d}\right)+\left(\Theta_{o k}^{d}, E_{o k}^{d}\right) .
\end{gathered}
$$

The first three terms are expressed in an explicit form, and the fourth terms represent the continued components, which are calculated by the quadrature method. Let us present expressions for the components of the functionals (19) and (20).

If $z \in[0, h]$ and $s \in \Omega$ component of the functional (19)

$$
\left(\Theta_{a}^{s}, E_{a}^{s}\right)\left(z, s^{-}\right)=
$$

$$
=E_{a}^{s}\left(s_{0} ; s^{-}\right) \exp \left\{-[\tau(h)-\tau(z)] / \mu_{0}\right\}
$$

is identical to the solution of the problem (5) for the direct solar flux reflected in the atmosphere from the interface: 


$$
\Phi_{0}\left(s_{0} ; z, s^{-}\right)=\left(\Theta_{a}^{s}, E_{a}^{s}\right) .
$$

The second component of the functional (19) is a continued diffuse component:

$$
\left(\Theta_{a}^{d}, E_{a}^{s}\right)(z, s)=E_{a}^{s}\left(s_{0} ; s_{0}^{-}\right) \Theta_{a}^{d}\left(s_{0}^{-} ; z, s\right) .
$$

The third component of the functional (19)

$$
\begin{gathered}
\left(\Theta_{a}^{s}, E_{a}^{d}\right)\left(z, s^{-}\right)= \\
=E_{a}^{d}\left(s^{-}\right) \exp \left\{-[\tau(h)-\tau(z)] /\left|\mu^{-}\right|\right\}
\end{gathered}
$$

represents the direct radiation traveling from the boundary $Z=h$ (a continued function).

The fourth component of the functional (19) is calculated taking into account the azimuth symmetry of the integrands, which are continued functions of angular arguments

$$
\begin{gathered}
E_{a}^{d}(\varphi)=E_{a}^{d}(2 \pi-\varphi)=E_{a}^{d}(-\varphi), \\
\Theta_{a}^{d}(\varphi)=\Theta_{a}^{d}(2 \pi-\varphi)=\Theta_{a}^{d}(-\varphi)
\end{gathered}
$$

and using the quadrature-method formula

$$
\begin{gathered}
\left(\Theta_{a}^{d}, E_{a}^{d}\right)(z, s)= \\
=\frac{1}{2 \pi} \int_{0}^{\pi} d \varphi_{h}^{-} \int_{-1}^{0}\left[\Theta_{a}^{d}\left(\mu_{h}^{-}, 0 ; z, \mu, \varphi-\varphi_{h}^{-}\right)+\right. \\
\left.+\Theta_{a}^{d}\left(\mu_{h}^{-}, 0 ; z, \mu, \varphi+\varphi_{h}^{-}\right)\right] E_{a}^{d}\left(\mu_{h}^{-}, \varphi_{h}^{-}\right) d \mu_{h}^{-} .
\end{gathered}
$$

If $Z \in[h, H]$ and $\widetilde{s} \in \Omega$ the singular component of the functional (20)

$$
\begin{gathered}
\left(\Theta_{o k}^{s}, E_{o k}^{s}\right)\left(\tilde{z}, \tilde{s}^{+}\right)= \\
=E_{o k}^{s}\left(\bar{s}_{0} ; \tilde{s}^{+}\right) \exp \left[-\tau(\tilde{z}) / \bar{\mu}_{0}\right]
\end{gathered}
$$

represents the direct solar flux in the ocean (6):

$$
\Phi^{0}\left(\bar{S}_{0} ; z, \bar{s}^{+}\right)=\left(\Theta_{o k}^{s}, E_{o k}^{s}\right) .
$$

The second component of the functional (20) is a continued component describing the contribution of the direct solar flux multiply scattered in the ocean:

$$
\left(\Theta_{o k}^{d}, E_{o k}^{s}\right)(\tilde{z}, \widetilde{s})=E_{o k}^{s}\left(\bar{s}_{0} ; \bar{s}_{0}^{-}\right) \Theta_{o k}^{d}\left(\bar{\mu}_{0}, 0 ; \tilde{z}, \widetilde{s}\right) .
$$

The third component of the functional (20)

$$
\left(\Theta_{o k}^{s}, E_{o k}^{d}\right)\left(\tilde{z}, \tilde{s}^{+}\right)=E_{o k}^{d}\left(\tilde{s}^{+}\right) \exp \left[-\tau(\tilde{z}) / \tilde{\mu}_{h}^{+}\right]
$$

represents the direct radiation that travels from the boundary and is caused by the diffuse irradiance component. The fourth component of the functional (20) is calculated by the quadrature method with allowance for the azimuth properties:

$$
\begin{gathered}
\left(\Theta_{o k}^{d}, E_{o k}^{d}\right)(\tilde{z}, \tilde{s})= \\
=\frac{1}{2 \pi} \int_{0}^{\pi} d \tilde{\varphi}_{h}^{+} \int_{0}^{1}\left[\Theta_{o k}^{d}\left(\tilde{\mu}_{h}^{+}, 0 ; \tilde{z}, \tilde{\mu}, \tilde{\varphi}-\tilde{\varphi}_{h}^{+}\right)+\right. \\
\left.+\Theta_{o k}^{d}\left(\tilde{\mu}_{h}^{+}, 0 ; \tilde{z}, \tilde{\mu}, \tilde{\varphi}+\tilde{\varphi}_{h}^{+}\right)\right] E_{o k}^{d}\left(\tilde{\mu}_{h}^{+}, \tilde{\varphi}_{h}^{+}\right) d \tilde{\mu}_{h}^{+} .
\end{gathered}
$$

\subsection{Structure of the OAS radiation field}

The solution of the initial problem (1) is usually sought as a superposition

$$
\Phi=\Phi^{0}+\Phi_{a}+\Phi_{d}+\Phi_{q} .
$$

The direct solar flux $\Phi^{0}$ in the atmosphere is determined by the solution to the Cauchy problem

$$
D \Phi^{0}=0,\left.\quad \Phi^{0}\right|_{t}=\pi S_{\lambda} \delta\left(s-s_{0}\right),\left.\Phi^{0}\right|_{d 1}=0
$$

in the explicit form (4).

The background (diffuse) solar radiation multiply scattered in the atmosphere $\Phi_{a}$ is found as the solution of the FBPA with nonreflecting boundaries

$$
K \Phi_{a}=S \Phi^{0},\left.\Phi_{a}\right|_{t}=0,\left.\Phi_{a}\right|_{d 1}=0 .
$$

The effect of the bottom in the layer $z \in[0, H]$ is described by the GBPOAS with the source $E_{H}=R_{H} \Phi_{d}$ :

$$
\begin{gathered}
K \Phi_{q}=0,\left.\Phi_{q}\right|_{t}=0,\left.\quad \Phi_{q}\right|_{b}=R_{H} \Phi_{q}+E_{H}, \\
\left.\Phi_{q}\right|_{d 1}=R_{1} \Phi_{q}+T_{21} \Phi_{q},\left.\Phi_{q}\right|_{d 2}=R_{2} \Phi_{q}+T_{12} \Phi_{q} .
\end{gathered}
$$

If the bottom is Lambertian and its albedo is $q$, then

$$
\Phi_{q}=q E_{H} \Psi /\left(1-q c_{0}\right), c_{0}=R_{H} \Psi,
$$

where the influence function of the bottom $\Psi(z, s)$ is the solution of the problem

$$
\begin{gathered}
K \Psi=0,\left.\Psi\right|_{t}=0,\left.\quad \Psi\right|_{b}=1, \\
\left.\Psi\right|_{d 1}=R_{1} \Psi+T_{21} \Psi,\left.\quad \Psi\right|_{d 2}=R_{2} \Psi+T_{12} \Psi,
\end{gathered}
$$

which reduces to a problem similar to (1.7).

The component $\Phi_{d}$, which takes into account the contribution of the ocean as both a reflecting underlying surface and a scattering medium, can be written in different ways depending on the model chosen for the 
structuring and estimation of mechanisms involved in the formation of the OAS radiation field.

\section{Mathematical model of radiation transfer in the ocean-atmosphere system with clouds}

Boundary-value problem for multi-layer heterogeneous system is considered

$$
K \Phi=F^{i n},\left.\Phi\right|_{t \downarrow}=F_{t}^{\downarrow},\left.\Phi\right|_{b \uparrow}=R_{b}^{\uparrow} \Phi+F_{b}^{\uparrow},
$$

at internal boundaries $h_{m}$ for $m=2 \div M$

$$
\begin{gathered}
\left.\Phi\right|_{d \uparrow, m}=\varepsilon\left(R_{m}^{\uparrow} \Phi+T_{m}^{\uparrow} \Phi\right)+F_{m-1}^{\uparrow}, \\
\left.\Phi\right|_{d \downarrow, m}=\varepsilon\left(R_{m}^{\downarrow} \Phi+T_{m}^{\downarrow} \Phi\right)+F_{m}^{\downarrow} ;
\end{gathered}
$$

integro-differential operator $K \equiv D-S$ with the linear operators: transfer operator $D$ and the collision integral $S ; m=1 \div M-$ number of layer.

Radiation propagation through the internal boundaries between the layers is described by equalmeasure restricted operators of reflectivity $R_{m}^{\downarrow}, R_{m}^{\uparrow}$ and transmittance $T_{m}^{\downarrow}, T_{m}^{\uparrow}$.

We seek solution in the form of a regular perturbation series

$$
\Phi=\sum_{n=0}^{\infty} \varepsilon^{n} \Phi^{(n)}
$$

Let`s introduce the algebraic vectors with the dimension $2 M$ :

$n$ - approximation of the solution

$$
\begin{aligned}
& \Phi^{(n)}=\left\{\Phi_{1}^{\downarrow(n)}, \Phi_{1}^{\uparrow(n)}, \Phi_{2}^{\downarrow(n)}, \Phi_{2}^{\uparrow(n)},\right. \\
& \left.\ldots, \Phi_{m}^{\downarrow(n)}, \Phi_{m}^{\uparrow(n)}, \ldots, \Phi_{M}^{\downarrow(n)}, \Phi_{M}^{\uparrow(n)}\right\} ;
\end{aligned}
$$

the complete solution

$$
\Phi=\left\{\Phi_{1}^{\downarrow}, \Phi_{1}^{\uparrow}, \Phi_{2}^{\downarrow}, \Phi_{2}^{\uparrow}, \ldots, \Phi_{m}^{\downarrow}, \Phi_{m}^{\uparrow}, \ldots, \Phi_{M}^{\downarrow}, \Phi_{M}^{\uparrow}\right\} ;
$$

$n$ - approximation of the sources

$$
\begin{aligned}
& F^{(n)}=\left\{F_{1}^{\downarrow(n)}, F_{1}^{\uparrow(n)}, F_{2}^{\downarrow(n)}, F_{2}^{\uparrow(n)},\right. \\
& \left.\ldots, F_{m}^{\downarrow(n)}, F_{m}^{\uparrow(n)}, \ldots, F_{M}^{\downarrow(n)}, F_{M}^{\uparrow(n)}\right\} ;
\end{aligned}
$$

the influence functions of the layers

$$
\Theta=\left\{\Theta_{1}^{\downarrow}, \Theta_{1}^{\uparrow}, \Theta_{2}^{\downarrow}, \Theta_{2}^{\uparrow}, \ldots, \Theta_{m}^{\downarrow}, \Theta_{m}^{\uparrow}, \ldots, \Theta_{M}^{\downarrow}, \Theta_{M}^{\uparrow}\right\} ;
$$

the initial approximation of the sources

$$
E=\left\{E_{1}^{\downarrow}, E_{1}^{\uparrow}, E_{2}^{\downarrow}, E_{2}^{\uparrow}, \ldots, E_{m}^{\downarrow}, E_{m}^{\uparrow}, \ldots, E_{M}^{\downarrow}, E_{M}^{\uparrow}\right\} ;
$$

the "scenario" on boundaries

$$
Z=\left\{Z_{1}^{\downarrow}, Z_{1}^{\uparrow}, Z_{2}^{\downarrow}, Z_{2}^{\uparrow}, \ldots, Z_{m}^{\downarrow}, Z_{m}^{\uparrow}, \ldots, Z_{M}^{\downarrow}, Z_{M}^{\uparrow}\right\}
$$

We produce decomposition of the original problem at $2 M$ problems with their own boundary conditions. The initial approximation - radiation from sources without radiation interchange between layers for $m=1 \div M$ :

$$
\begin{gathered}
K \Phi_{m}^{\downarrow(0)}=F_{m}^{\downarrow_{i n}},\left.\Phi_{m}^{\downarrow(0)}\right|_{d \downarrow, m}=F_{m}^{\downarrow},\left.\Phi_{m}^{\downarrow(0)}\right|_{d \uparrow, m+1}=0 ; \\
K \Phi_{m}^{\uparrow(0)}=F_{m}^{\uparrow i n},\left.\Phi_{m}^{\uparrow(0)}\right|_{d \downarrow, m}=0,\left.\Phi_{m}^{\uparrow(0)}\right|_{d \uparrow, m+1}=F_{m}^{\uparrow} .
\end{gathered}
$$

The approximation $n \geq 1$ - system of $2 M$ equations for layers $m=1 \div M$ :

$$
\begin{gathered}
K \Phi_{m}^{\downarrow(n)}=0,\left.\Phi_{m}^{\downarrow(n)}\right|_{d \downarrow, m}=F_{m}^{\downarrow(n-1)},\left.\Phi_{m}^{\downarrow(n)}\right|_{d \uparrow, m+1}=0 ; \\
K \Phi_{m}^{\uparrow(n)}=0,\left.\Phi_{m}^{\uparrow(n)}\right|_{d \downarrow, m}=0,\left.\Phi_{m}^{\uparrow(n)}\right|_{d \uparrow, m+1}=F_{m}^{\uparrow(n-1)}
\end{gathered}
$$

with sources at the boundaries $h_{m}$ for $m=1 \div M+1$ :

$$
\begin{gathered}
F_{m}^{\downarrow(n)}=T_{m}^{\downarrow} \Phi_{m-1}^{\downarrow(n)}+T_{m}^{\downarrow} \Phi_{m-1}^{\uparrow(n)}+R_{m}^{\downarrow} \Phi_{m}^{\downarrow}+R_{m}^{\downarrow} \Phi_{m}^{\uparrow(n)} ; \\
F_{m}^{\uparrow(n)}=R_{m+1}^{\uparrow} \Phi_{m}^{\downarrow}+R_{m+1}^{\uparrow} \Phi_{m}^{\uparrow(n)}+ \\
+T_{m+1}^{\uparrow} \Phi_{m+1}^{\downarrow \downarrow(n)}+T_{m+1}^{\uparrow} \Phi_{m+1}^{\uparrow(n)} .
\end{gathered}
$$

Solution is assumed in the form of linear functionals for each layer with $m=1 \div M$ :

$$
\Phi_{m}^{\downarrow(n)}=\left(\Theta_{m}^{\downarrow}, F_{m}^{\downarrow(n-1)}\right) ; \quad \Phi_{m}^{\uparrow(n)}=\left(\Theta_{m}^{\uparrow}, F_{m}^{\uparrow(n-1)}\right) .
$$

Functionals kernels - influence functions of layers with $m=1 \div M$ are determined from boundary-value problems

$$
\begin{gathered}
K \Theta_{m}^{\downarrow}=0,\left.\quad \Theta_{m}^{\downarrow}\right|_{d \downarrow, m}=f_{\delta, m}^{\downarrow}, \\
\left.\Theta_{m}^{\downarrow}\right|_{d \uparrow, m+1}=0 ; \quad f_{\delta, m}^{\downarrow}=\delta\left(s-s_{m}^{\downarrow}\right) ; \\
K \Theta_{m}^{\uparrow}=0,\left.\quad \Theta_{m}^{\uparrow}\right|_{d \downarrow, m}=0, \\
\left.\Theta_{m}^{\uparrow}\right|_{d \uparrow, m+1}=f_{\delta, m}^{\uparrow} ; \quad f_{\delta, m}^{\uparrow}=\delta\left(s-S_{m}^{\uparrow}\right) .
\end{gathered}
$$

The components of vectorial linear functional are computed for $z \in\left[h_{m}, h_{m+1}\right]$ by formulas: 


$$
\begin{aligned}
& \left(\Theta_{m}^{\downarrow}, f_{m}^{\downarrow}\right)(z, s)=\int_{\Omega^{+}} \Theta_{m}^{\downarrow}\left(s_{m}^{\downarrow} ; z, s\right) f_{m}^{\downarrow}\left(h_{m}, s_{m}^{\downarrow}\right) d s_{m}^{\downarrow} ; \\
& \left(\Theta_{m}^{\uparrow}, f_{m}^{\uparrow}\right)(z, s)=\int_{\Omega^{-}} \Theta_{m}^{\uparrow}\left(s_{m}^{\uparrow} ; z, s\right) f_{m}^{\uparrow}\left(h_{m+1}, s_{m}^{\uparrow}\right) d s_{m}^{\uparrow},
\end{aligned}
$$

where $f_{m}^{\downarrow}\left(h_{m}, s_{m}^{\downarrow}\right)$ is the brightness source distribution at boundary $Z=h_{m}$ when $s_{m}^{\downarrow} \in \Omega^{\downarrow} ; f_{m}^{\uparrow}\left(h_{m+1}, s_{m}^{\uparrow}\right)$ is the brightness source distribution at boundary $Z=h_{m+1}$ when $s_{m}^{\uparrow} \in \Omega^{\uparrow}$.

\section{Results}

To calculate the total radiation inside or on the boundaries of the system with radiation exchange between the layers the vectorial optical transfer operator (VOTO) is constructed by the influence functions method. The kernels of operators are the influence functions of the layers. Operators of radiation transmittance and reflectance on the boundaries between the layers are formulated based on the collision integrals, and the separate layers are united in a system by these operators.

The $\boldsymbol{n}$-approximation of solution is stated in form of vectorial linear functional

$$
\Phi^{(n)}=\left(\Theta, F^{(n-1)}\right)
$$

where sources in $(n-1)$-approximation $F=P \Phi^{(n-1)}$. Two sequential $n$-approximations are connected by recursion relation

$$
\Phi^{(n)}=\left(\Theta, P \Phi^{(n-1)}\right)
$$

where $P$ - matrix of band type with layers reflection and trasmission characteristics. The notion can be found

$$
\Phi^{(n)}=\left(\Theta, G^{n-1} E\right)
$$

where $E-$ initial approximation. Matrix-vector operation, which describe one act of radiation interaction with internal and external boundaries and take into account multiply scattering in the layers through influence functions, is determined:

$$
G F=P(\Theta, F)=
$$

Asymptotical exact solution is obtained in the form of vectorial linear functional - optical transfer operator:

$$
\Phi=(\Theta, Z) .
$$

$$
\left[\begin{array}{l}
0 \\
R_{2}^{\uparrow}\left(\Theta_{1}^{\downarrow}, F_{1}^{\downarrow}\right)+R_{2}^{\uparrow}\left(\Theta_{1}^{\uparrow}, F_{1}^{\uparrow}\right)+ \\
+T_{2}^{\uparrow}\left(\Theta_{2}^{\downarrow}, F_{2}^{\downarrow}\right)+T_{2}^{\uparrow}\left(\Theta_{2}^{\uparrow}, F_{2}^{\uparrow}\right) \\
\mathrm{M} \\
T_{m}^{\downarrow}\left(\Theta_{m-1}^{\downarrow}, F_{m-1}^{\downarrow}\right)+T_{m}^{\downarrow}\left(\Theta_{m-1}^{\uparrow}, F_{m-1}^{\uparrow}\right)+ \\
+R_{m}^{\downarrow}\left(\Theta_{m}^{\downarrow}, F_{m}^{\downarrow}\right)+R_{m}^{\downarrow}\left(\Theta_{m}^{\uparrow}, F_{m}^{\uparrow}\right) \\
R_{m+1}^{\uparrow}\left(\Theta_{m}^{\downarrow}, F_{m}^{\downarrow}\right)+R_{m+1}^{\uparrow}\left(\Theta_{m}^{\uparrow}, F_{m}^{\uparrow}\right)+ \\
+T_{m+1}^{\uparrow}\left(\Theta_{m+1}^{\downarrow}, F_{m+1}^{\downarrow}\right)+T_{m+1}^{\uparrow}\left(\Theta_{m+1}^{\uparrow}, F_{m+1}^{\uparrow}\right) \\
\mathrm{M} \\
T_{M}^{\downarrow}\left(\Theta_{M-1}^{\downarrow}, F_{M-1}^{\downarrow}\right)+T_{M}^{\downarrow}\left(\Theta_{M-1}^{\uparrow}, F_{M-1}^{\uparrow}\right)+ \\
+R_{M}^{\downarrow}\left(\Theta_{M}^{\downarrow}, F_{M}^{\downarrow}\right)+R_{M}^{\downarrow}\left(\Theta_{M}^{\uparrow}, F_{M}^{\uparrow}\right) \\
R_{b}^{\uparrow}\left(\Theta_{M}^{\downarrow}, F_{M}^{\downarrow}\right)+R_{b}^{\uparrow}\left(\Theta_{M}^{\uparrow}, F_{M}^{\uparrow}\right)
\end{array}\right]
$$

"Scenario" - vector $Z$ distribution of brightness on internal and external boundaries $m=1 \div M+1$ :

$$
Z \equiv Y E \equiv \sum_{n=0}^{\infty} G^{n} E=\sum_{n=0}^{\infty} P \Phi^{(n)}
$$

is sum of Neyman series by multiplicity passing of radiation through boundaries with consideration of multiply scattering contribution by the help of influence functions of each layer

Original mathematical tools are proposed for the first time to model radiation transfer in multi-layer nonhomogenous heterogenic natural media with different radiation regimes within separate regions of the system. "Free atmosphere - multi-layer cloudiness - near surface layer of the atmosphere - ocean" system is heterogenic, in particular. The proposed approach is based on construction of generalized solutions in the form of matrix functionals; vectors of influence functions for each layer of the system serve as kernels of the functionals. The influence functions of the layers with different aerosol and molecular characteristics of scattering and absorption and radiation regimes can thus be calculated by various methods in different approaches of radiation transfer theory.

This approach enables to simulate radiation fields in a wide range of variations of the optical characteristics of these layers and to analyze mechanisms of radiation characteristics formation inside and outside the layers as well as to estimate any contribution of each region. Reflected radiation and distributions of radiation characteristics inside the layer near to the border illuminated by an external flux are thus calculated with higher accuracy than in common-used techniques.

The boundary problem for each layer can be solved depending on optical thickness, scattering and absorption characteristics by one of the following techniques: 
i) as a solution of transfer equation with an azimuth dependence;

ii) as a solution of the problem with azimuth symmetry;

iii) as a solution in two-flux approach;

iv) as an approximate solution in asymptotic approach.

Operators of radiation transmittance and reflectance on the boundaries between the layers are formulated based on the collision integrals and the separate layers are united in a system by these operators. To calculate the total radiation inside or on the boundaries of the system with radiation exchange between the layers a matrix-vector operation is constructed for the operators the kernels of which are given by the influence functions of the layers.

The representation of the solution to boundary-value problem as a functional is the optical transfer operator of the radiation transfer system which establishes the explicit relationship between the recording radiation and the "scenarios" (the optical image) at the dividing boundaries of media. In turn, by the use of the influence functions, the "scenarios" is described clearly through the characteristics of the reflection and transmission of the dividing boundaries at the given its illumination. The influence functions are invariant about the conditions of the illumination and the properties of the dividing boundaries.

\section{Conclusion}

On a planetary scale, there is an urgent problem of creating international global monitoring of the Earth with the aim of studying its evolution and predicting natural disasters and anthropogenic-technogenic catastrophic processes, as well as environmental and climate changes on a local and global scale.

The theoretical basis for estimating radiation forcing is the solution of direct and inverse problems of the radiation transfer theory [8, 9]. Hyperspectral remote sensing of the Earth is the most important technology for monitoring and evaluating the radiation impact on the planet Earth. In recent years, close attention of researchers have focused on improving and developing methods and tools for more thorough and reliable accounting of the absorption of major and minor gases in the atmosphere and assessing the influence of the absorbing factor on radiation forcing.

The Table shows data on the number of spectral lines of absorbing gases in the most complete and reliable version of the HITRAN-2016 database (USA).

Table of spectral lines

\begin{tabular}{|c|c|}
\hline Molecule & Sum of lines for all isotopes \\
\hline $\mathrm{H}_{2} \mathrm{O}$ & 313787 \\
\hline $\mathrm{CO}_{2}$ & 559874 \\
\hline $\mathrm{O}_{3}$ & 449570 \\
\hline $\mathrm{N}_{2} \mathrm{O}$ & 160287 \\
\hline
\end{tabular}

\begin{tabular}{|c|l|}
\hline $\mathrm{CO}$ & 5381 \\
\hline $\mathrm{CH}_{4}$ & 450332 \\
\hline $\mathrm{O}_{2}$ & 14085 \\
\hline Total & 1953316 \\
\hline
\end{tabular}

Scientists from different countries are involved in the formation of these databases [11]. Our team members are also engaged in analysis, "reference calculations" and applications of these databases to calculations [12]. According to 2016 data, about 2 million absorption lines are already known, and this does not take into account the continuous absorption of water in different phases (water vapor, droplets, ice crystals) and aerosols of different origin. To calculate the absorption coefficients, profiles of meteorological data (temperature, pressure) and component concentrations are required. And, of course, important times of day, seasons, regions, etc. And, of course, you need to take into account the diversity of underlying surfaces and the influence of oceans and clouds.

\section{Acknowledgements}

The article is dedicated to the memory of our teachers. Three Russian Geniuses - "Three K" - Mstislav Vsevolodovich Keldysh (10.02.1911-24.06.1978) Chief Cosmonautics Theorist, Three times Hero of Socialist Labor (1956, 1961, 1971); Sergey Pavlovich Korolev (12.01.1907-14.01.1966) - Chief Designer of Cosmonautics, Twice Hero of Socialist ILbor (1956, 1961); Igor Vasilyevich Kurchatov (12.01.190307.02.1960) - "Father of the Russian atomic bomb", Three Times Hero of Socialist Labor (1949, 1951, 1954) - they headed and made the scientific and technological revolution in the Union of Soviet Socialist Republics (USSR) in the middle of the XX-th century, and their names forever entered the history of the world mankind civilization , the USSR and Russia. It is thanks to their scientific exploits and merits that the strategic parity of the two political systems and military blocs led by USSR)and USA was ensured, and for almost 75 years there are no global world wars on the planet.

This research was supported by the State project 0017-2019-0002.

\section{References}

1. V.E. Ryabinin, V.I. Sychev, Uchenye zapiski RSHU, 54, 73-79 (2019)

2. M.V. Keldysh, Creative portrait on the memoirs of contemporaries (Nauka, Moscow, 2001)

3. T.A. Sushkevich, Jr. Rse. Cosmos, 8(1), 9-25 (2011)

4. T.A. Sushkevich, Jr. Rse. Cosmos, 8(4), 9-22 (2011)

5. A.S. Monin, Yu.A. Shishkov, UFN, 170(4), 419445 (2000)

6. R.I. Nigmatulin, Expert, 34, 46-51 (2018) 
7. T.A. Sushkevich, Fundam. Prikl. Gidrofiz., 13, 103-109 (2020)

8. T.A. Sushkevich, S.A. Strelkov, Characteristics Method in Atmospheric Optics Problems (Nauka, Moscow, 1990)

9. T.A. Sushkevich, Mathematical Models of Radiation Transfer (BINOM, Laboratory of Knowledge, Moscow, 2005)

10. T.A. Sushkevich, A.A. Ioltukhovsky, Preprint KIAM RAS, 9 (1986)

11. I.E. Gordon, L.S. Rothman, C. Hill et al., JQSRT 203, 3-69 (2017)

12. B.A. Fomin, G.E. Kolokutin, Jr. Rse. Cosmos 16(3), 17-24 (2019)

13. C.D. Mobley, B. Gentili, H.R. Gordon, Z. Jin, G.W. Kattawar, A. Morel, Appl. Opt., 32, 74847504 (1993)

14. Z. Jin, K. Stanmes, Appl. Opt., 33, 431-443 (1994)

15. J. Lenoble, Radiative transfer in scattering and absorbing atmospheres: standard computational procedures (A. DEEPAK Publishing, Hampton, Virginia, USA, 1985). 